\title{
Charge transfer energies of benzene physisorbed on a graphene sheet from constrained density functional theory
}

\author{
Subhayan Roychoudhury, Carlo Motta, and Stefano Sanvito \\ School of Physics, AMBER and CRANN Institute, Trinity College Dublin, Dublin 2, Ireland
}

(Received 2 November 2015; published 22 January 2016)

\begin{abstract}
Constrained density functional theory (CDFT) is used to evaluate the energy level alignment of a benzene molecule as it approaches a graphene sheet. Within CDFT the problem is conveniently mapped onto evaluating total energy differences between different charge-separated states, and it does not consist in determining a quasiparticle spectrum. We demonstrate that the simple local density approximation provides a good description of the level alignment along the entire binding curve, with excellent agreement to experiments at an infinite separation and to $G W$ calculations close to the bonding distance. The method also allows us to explore the effects due to the presence of graphene structural defects and of multiple molecules. In general, all our results can be reproduced by a classical image charge model taking into account the finite dielectric constant of graphene.
\end{abstract}

DOI: 10.1103/PhysRevB.93.045130

\section{INTRODUCTION}

Organic molecular crystals, namely crystals composed of organic molecules held together by weak van der Waals forces, are emerging as excellent candidates for fabricating nanoscale devices. These have potential application in electronics and optoelectronics, in particular, in areas such as solar energy harvesting, surface photochemistry, organic electronics, and spintronics [1-5]. A feature common to such class of devices is that they are composed from both an organic and inorganic component, where the first forms the active part of the device and the second provides the necessary electrical contact to the external circuitry. Clearly the electronic structure of the interface between these two parts plays a crucial role in determining the final device performance and needs to be understood carefully. In particular, it is important to determine how charge transfers between the organic and the inorganic component and the energies at which the transfer takes place. This is a challenging task, especially in the single-molecule limit. Upon adsorption on a substrate, the electron addition and removal energies of a molecule change value from that of their gas phase counterparts. This is expected since, when the molecule is physisorbed on a polarizable substrate, the removal (addition) of an electron from (to) the molecule gives rise to a polarization of the substrate. The image charge accumulated on the substrate in the vicinity of the molecule alters the addition or removal energy of charge carriers from the molecule.

A common way to calculate the addition and removal energies is to use a quasiparticle (QP) description. Within the QP picture, one ignores the effects of relaxation of molecular orbitals due to addition or removal of electrons and consequently takes the relative alignment of the metal Fermi level $E_{\mathrm{F}}$ with either the lowest unoccupied molecular orbital (LUMO) or highest occupied molecular orbital (HOMO) of a molecule as removal energy. This effectively corresponds to associate the electron affinity and the ionization potential, respectively, to the LUMO and HOMO of the molecule. The adequacy of the QP description then depends on the level of theory used to calculate the energy levels of the HOMO and LUMO.
If the theory of choice is density functional theory (DFT) [6], then a number of observations should be made. First, it is important to note that except for the energy of the HOMO, which can be rigorously interpreted as the negative of the ionization potential [7], in general the Kohn-Sham orbitals cannot be associated to QP energies. This is, however, commonly done in practice and often the Kohn-Sham QP levels provide a good approximation to the true removal energies, in particular, in the case of metals. For molecules unfortunately the situation is less encouraging with the local and semilocal approximations of the exchange and correlation functional, namely the local density approximation (LDA) and the generalized gradient approximation (GGA), performing rather poorly even for the HOMO level. Such a situation is partially corrected by hybrid functionals [8] or by a functional explicitly including self-interaction corrections $[9,10]$, and extremely encouraging results have been recently demonstrated for range separated functionals $[11,12]$.

The calculation of the energy level alignment of a molecule in the proximity of a metal, however, presents additional problems. In fact, the formation of the image charge, although it is essentially a classical electrostatic phenomenon, has a completely nonlocal nature. This means that unless a given functional is explicitly nonlocal it will in general fail in capturing such an effect. The most evident feature of such failure is that the position of the HOMO and LUMO changes very little when a molecule approaches a metallic surface [13]. Such failure is typical of the LDA and GGA, and both hybrid and self-interaction corrected functionals do not improve much the situation. A possible solution to the problem is that of using an explicit many-body approach to calculate the QP spectrum. This is for instance the case of the $G W$ approximation [14], which indeed is capable of capturing the energy levels renormalization due to the image charge effect [15]. The $G W$ scheme, however, is highly computationally demanding and can be applied only to rather small systems. This is not the case for molecules on surfaces, where the typical simulation cells have to include several atomic layers of the metal and they should be laterally large enough to contain the image charge in full. This, in addition to the $G W$ necessity to 
compute a significant fraction of the empty states manifold, make the calculations demanding and it is often not simple even to establish whether convergence has been achieved.

In this paper we approach the problem of evaluating the charge transfer energies of an organic molecule physisorbed on an inorganic substrate with the help of a much more resourceefficient alternative, namely constrained density functional theory (CDFT) [16]. In CDFT one transfers one electron from the molecule to the substrate (and vice versa) and calculates the difference in energy with respect to the locally charge neutral configuration (no excess of charge either on the molecule or the substrate) [17]. As such, CDFT avoids the calculation of a QP spectrum, which is instead replaced by a series of total energy calculations for different charge distributions. This approach is free of any interpretative issues and benefits from the fact that even at the LDA level the total energy is usually an accurate quantity. Finally, it is important to remark that, for any given functional, CDFT is computationally no more demanding than a standard DFT calculation, so that both the LDA and the GGA allow one to treat large systems and to monitor systematically the approach to convergence.

Here we use the CDFT approach to study the adsorption of molecules on a two-dimensional (2D) metal in various configurations. It must be noted that in contrast to a regular three-dimensional (3D) metal, in a 2D one the image charge induced on the substrate is constrained within a one-atom thick sheet. This means that electron screening is expected to be less efficient than in a standard 3D metal and the features of the image charge formation in general more complex. In particular, we consider here the case of graphene, whose technological relevance is largely established [18]. Most importantly for our work, recently graphene has been used as a template layer for the growth of organic crystals [19]. It is then quite important to understand how such a template layer affects the level alignment of the molecules with the metal. As a model system we consider a simple benzene molecule adsorbed on a sheet of graphene. This has been studied in the past [20-22], so that a good description of the equilibrium distance and the corresponding binding energy of the molecule in various configurations with respect to the graphene sheet are available. Furthermore, a $G_{0} W_{0}$ study for some configurations exists [23], so that our calculated QP gap can be benchmarked.

Our calculations show that the addition and removal energies decrease in absolute value as the molecule is brought closer to the graphene sheet. Such decrease can be described with a classical electrostatic model taking into account the true graphene dielectric constant. As it will be discussed, a careful choice of the substrate unit cell is necessary to ensure the inclusion of the image charge, whose extension strongly depends on the molecule-substrate distance. We also reveal that the presence of defects in the graphene sheet, such as a Stone-Wales one, does not significantly alter the charge transfer energies. In realistic situations, e.g., at the interface between a molecular crystal and an electrode, a molecule is surrounded by many others, which might alter the level alignment. We thus show calculations where neighboring molecules are included above, below, and in the same plane of the one under investigation. Interestingly, our results suggest that the charge transfer states are weakly affected by the presence of other molecules.

\section{METHOD}

In order to find the ground state energy of a system, KohnSham DFT minimizes a universal energy functional

$$
\begin{aligned}
E[\rho]= & \sum_{\sigma}^{\alpha, \beta} \sum_{i}^{N_{\sigma}}\left\langle\phi_{i \sigma}\left|-\frac{1}{2} \nabla^{2}\right| \phi_{i \sigma}\right\rangle+\int d \mathbf{r} v_{n}(\mathbf{r}) \rho(\mathbf{r}) \\
& +J[\rho]+E_{\mathrm{xc}}\left[\rho^{\alpha}, \rho^{\beta}\right],
\end{aligned}
$$

where $J, E_{\mathrm{xc}}$, and $v_{n}$ denote, respectively, the Hartree, exchange-correlation (XC), and external potential energies. The Kohn-Sham orbitals $\phi_{i \sigma}$ for an electron with spin $\sigma$ define the noninteracting kinetic energy $\left\langle\phi_{i \sigma}\left|-\frac{1}{2} \nabla^{2}\right| \phi_{i \sigma}\right\rangle$, while $N_{\sigma}$ is the total number of electrons with spin $\sigma$. The electron density is then given by $\rho(\mathbf{r})=\sum_{\sigma}^{\alpha, \beta} \rho^{\sigma}=$ $\sum_{\sigma} \sum_{i}^{N_{\sigma}}\left|\phi_{i \sigma}(\mathbf{r})\right|^{2}$.

In contrast to regular DFT, in CDFT one wants to find the ground state energy of the system subject to an additional constraint of the form

$$
\sum_{\sigma} \int w_{\mathrm{c}}^{\sigma}(\mathbf{r}) \rho^{\sigma}(\mathbf{r}) d \mathbf{r}=N_{\mathrm{c}}
$$

where $w_{\mathrm{c}}^{\sigma}$ is a weighting function that describes the spatial extension of the constraining region and $N_{\mathrm{c}}$ is the number of electrons that one wants to confine in that region. In our case $w_{\mathrm{c}}^{\sigma}(\mathbf{r})$ is set to 1 inside a specified region and zero elsewhere. In order to minimize $E[\rho]$ subject to the constraint, we introduce a Lagrange multiplier $V_{\mathrm{c}}$ and define the constrained functional [16]

$$
W\left[\rho, V_{\mathrm{c}}\right]=E[\rho]+V_{\mathrm{c}}\left(\sum_{\sigma} \int w_{\mathrm{c}}^{\sigma}(\mathbf{r}) \rho^{\sigma}(\mathbf{r}) d \mathbf{r}-N_{\mathrm{c}}\right) .
$$

Now the task is that of finding the stationary point of $W\left[\rho, V_{\mathrm{c}}\right]$ under the normalization condition for the KohnSham orbitals. This leads to a new set of Kohn-Sham equations

$$
\begin{aligned}
& {\left[-\frac{1}{2} \nabla^{2}+v_{n}(\mathbf{r})+\int \frac{\rho\left(\mathbf{r}^{\prime}\right)}{\left|\mathbf{r}-\mathbf{r}^{\prime}\right|} d \mathbf{r}^{\prime}+v_{\mathrm{xc}}^{\sigma}(\mathbf{r})+V_{\mathrm{c}} w_{\mathrm{c}}^{\sigma}(\mathbf{r})\right] \phi_{i \sigma}} \\
& \quad=\epsilon_{i \sigma} \phi_{i \sigma}
\end{aligned}
$$

where $v_{\mathrm{xc}}^{\sigma}(\mathbf{r})$ is the exchange and correlation potential. Equation (4) does not compute $V_{\mathrm{c}}$, which remains a parameter. However, for each value of $V_{c}$ it produces a unique set of orbitals corresponding to the minimum-energy density. In this sense we can treat $W\left[\rho, V_{\mathrm{c}}\right]$ as a functional of $V_{\mathrm{c}}$ only. It can be proved that $W\left[\rho, V_{\mathrm{c}}\right]$ has only one stationary point with respect to $V_{\mathrm{c}}$, where it is maximized [16]. Most importantly, the stationary point satisfies the constraint. One can then design the following procedure to find the stationary point of $W\left[\rho, V_{\mathrm{c}}\right]$ : (i) start with an initial guess for $\phi_{i \sigma}$ and $V_{c}$ and solve Eq. (4); (ii) update $V_{\mathrm{c}}$ until the constraint Eq. (2) is satisfied; and (iii) start over with the new $V_{\mathrm{c}}$ and a new set of $\phi$ s.

Here we use CDFT to calculate the charge transfer energy between a benzene molecule and a graphene sheet. For any given molecule-to-substrate distance $d$ we need to perform three different calculations:

(1) A regular DFT calculation in order to determine the ground state total energy $E_{0}(d)$ and the amount of charge on each subsystem (i.e., on the molecule and on the graphene sheet). 
(2) A CDFT calculation with the constraint that the graphene sheet contains one extra electron and the molecule contains one hole. This gives the energy $E_{+}(d)$.

(3) A CDFT calculation with the constraint that the graphene sheet contains one extra hole and the molecule one extra electron. This gives the energy $E_{-}(d)$.

The charge transfer energy for removing an electron from the molecule and placing it on the graphene sheet is then $E_{\mathrm{CT}}^{+}(d)=E_{+}(d)-E_{0}(d)$. Similarly, that for the transfer of an electron from the graphene sheet to the molecule is $E_{\mathrm{CT}}^{-}(d)=E_{0}(d)-E_{-}(d)$. Since in each run the cell remains charge neutral, there is no need here to apply any additional corrections. However, we have to keep in mind that this method is best used when the two subsystems are well separated so that the amount of charge localized on each subsystem is a well defined quantity.

In our calculations we use the CDFT implementation [17] for the popular DFT package SIESTA [24], which adopts a basis set formed by a linear combination of atomic orbitals (LCAO). The constrain is introduced in the form of a projection over a specified set of basis orbitals and in particular use the Lowdin projection scheme. Throughout this work we adopt a double-zeta polarized basis set with an energy cutoff of $0.02 \mathrm{Ry}$. The calculations are done with norm-conserving pseudopotential and the LDA is the exchange-correlation functional of choice. A mesh cutoff of $300 \mathrm{Ry}$ has been used for the real-space grid. We impose periodic boundary conditions with different cell sizes and the $k$-space grid is varied in accordance with the size of the unit cell. For instance, an in-plane $5 \times 5 k$ grid has been used for a $13 \times 13$ graphene supercell.

\section{RESULT AND DISCUSSION}

\section{A. Single benzene on pristine graphene}

We begin this section with a discussion on the equilibrium distance for a benzene molecule adsorbed on graphene. This is obtained by simply minimizing the total energy difference $\Delta E_{\mathrm{int}}=E_{\mathrm{Benz}+\mathrm{Gr}}-\left(E_{\mathrm{Benz}}+E_{\mathrm{Gr}}\right)$, where $E_{\mathrm{Benz}+\mathrm{Gr}}$ is the total energy for the cell containing benzene on graphene, while $E_{\mathrm{Benz}}\left(E_{\mathrm{Gr}}\right)$ is the total energy of the same cell when only the benzene (graphene) is present. This minimization is performed for two different orientations of the benzene molecule with respect to the graphene sheet: the hollow $(\mathrm{H})$ configuration, in which all the carbon atoms of the benzene ring are placed exactly above the carbon atoms of graphene, and the stack (S) configuration, in which alternate carbon atoms of the benzene molecule are placed directly above carbon atoms of the graphene sheet [see Figs. 1(a) and 1(b)]. For the $\mathrm{H}$ configuration we find an equilibrium distance of $3.4 \AA$, while for the $\mathrm{S}$ one this becomes $3.25 \AA$. These results are in fair agreement with another LDA theoretical study [22] (predicting 3.4 and $3.17 \AA$, respectively, for for the $\mathrm{H}$ and $\mathrm{S}$ orientations). Note that a more precise evaluation of such distances requires the use of van der Waals corrected functionals. This exercise, however, is outside the scope of our work and here we just wish to establish that the equilibrium distance is large enough for our constraint to remain well defined. It can also be noted that the equilibrium distance of $3.6 \AA$ obtained with a vdW-DF study [21] is not very different from our LDA result.

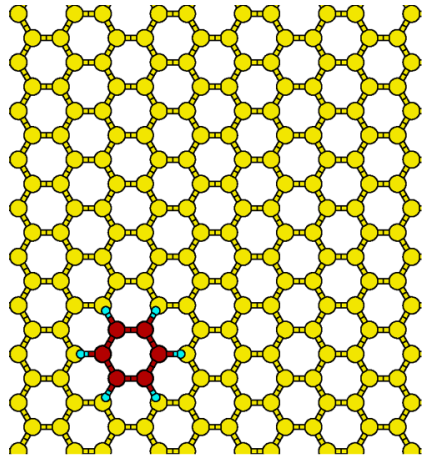

(a)

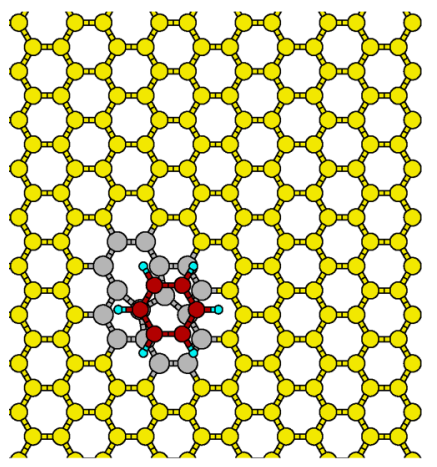

(c)

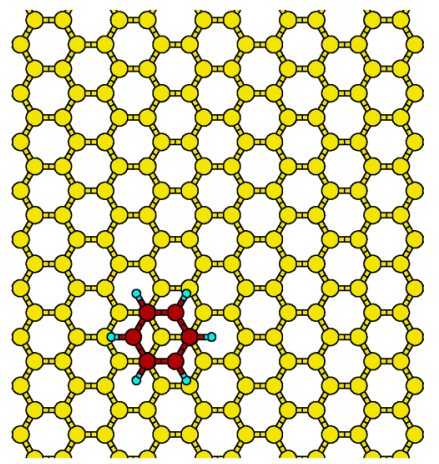

(b)

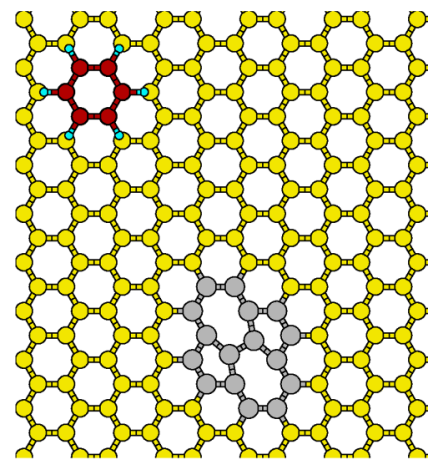

(d)
FIG. 1. Top view of the (a) $\mathrm{H}$, (b) $\mathrm{S}$, (c) $\mathrm{H}_{\mathrm{sw}-\mathrm{T}}$, and (d) $\mathrm{H}_{\mathrm{sw}-\mathrm{A}}$ configurations. Red spheres are carbon atoms belonging to benzene ring, while yellow spheres are those of the graphene sheet. In the graphene sheet with a SW defect, the carbon atoms in all rings affected by the deformation are marked in gray.

We then study the dependence of the charge transfer energies on the size of the graphene unit cell used. This is achieved by looking at the charge transfer gap $E_{\mathrm{CT}}^{+}(d)-E_{\mathrm{CT}}^{-}(d)$ as a function of the unit cell size at various molecule-to-graphene distances (see Fig. 2). When the molecule is very close to the graphene sheet, after transferring an electron, the image charge

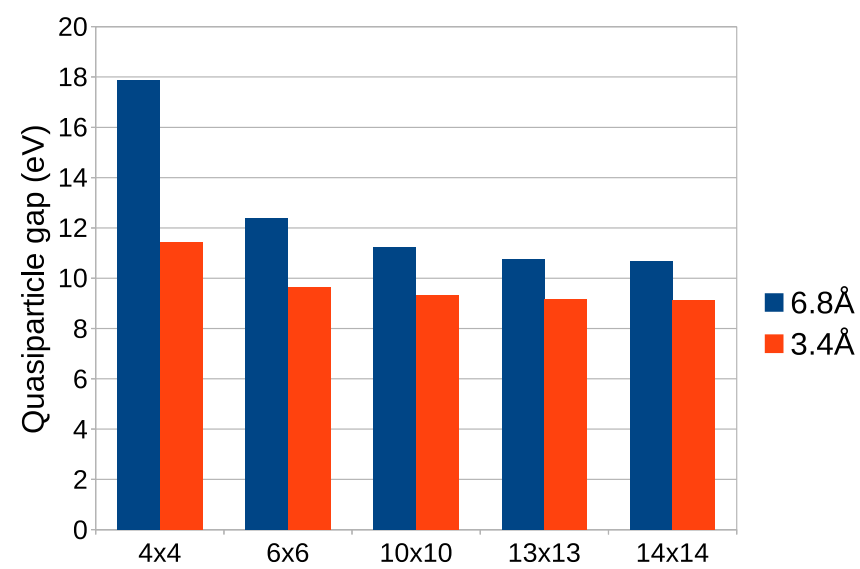

FIG. 2. Charge transfer energy gap $E_{\mathrm{CT}}^{+}-E_{\mathrm{CT}}^{-}$for different unit cell sizes of graphene sheet. The results are presented for two different molecule-to-graphene distances: 3.4 and $6.8 \AA$. 
is strongly attracted by the oppositely charged molecule and thereby remains highly localized. However, as the molecule moves away from the substrate, the attraction reduces since the Coulomb potential decays with distance, resulting in a delocalization of the image charge. This will eventually spread uniformly all over the graphene sheet in the limit of an infinite distance. If the unit cell is too small, the image charge will be artificially overconfined, resulting in an overestimation of $E_{-}(d)$ and $E_{+}(d)$ and, as a consequence, of the charge transfer energies. This effect can be clearly seen in Fig. 2, where we display the variation of the charge transfer energies as a function of the cell size. Clearly for the shorter distance (3.4 $\AA$ corresponding to the average equilibrium distance), the energy gap converges for supercells of about $10 \times 10(10 \times 10$ graphene primitive cells). At the larger distance of $6.8 \AA$ the same convergence is achieved for a $13 \times 13$ supercell.

Next we compute the charge transfer energies as a function of the distance between the sheet and the molecule. In order to compare our results with the gap expected in the limit of an infinite distance, we need to evaluate first the ionization potential $I_{\mathrm{MOL}}$ and the electron affinity $A_{\mathrm{MOL}}$ of the isolated benzene molecule. This is also obtained in terms of total energy differences between the neutral and the positively and negatively charged molecule, namely with the $\triangle \mathrm{SCF}$ method. This returns a quasiparticle energy gap $I_{\mathrm{MOL}}-A_{\mathrm{MOL}}$ of $11.02 \mathrm{eV}$, in good agreement (within $4.5 \%$ ) with the experimental value $[25,26]$. Likewise we also determine the Fermi level $\left(W_{\mathrm{F}}\right)$ of graphene, which is found to be $4.45 \mathrm{eV}$.

In Fig. 3(a) we show the change in the charge transfer energy gap with the distance of the benzene from the graphene sheet for the $\mathrm{H}$ configuration. As expected, when the molecule is close to the surface, there is a considerably large attraction between the image charge and the opposite charge excess on the molecule, resulting in an additional stabilization of the system and a reduction in magnitude of $E_{+}(d)$ and $E_{-}(d)$. Hence, in such a case the charge transfer energies have a reduced magnitude and the charge transfer gap is smaller than that in the gas phase. Then, as the molecule moves away from the graphene sheet, the charge transfer energies increase and so does the charge transfer energy gap until it eventually reaches the value corresponding to the HOMO-LUMO gap of the isolated molecule in the limit of an infinite distance. The same behavior is also found for the $\mathrm{S}$ configuration. Furthermore, note that, since the size of the image charge is essentially determined by the electron screening of the graphene sheet alone, the same cell size is required for both the $\mathrm{S}$ and $\mathrm{H}$ configurations.

In Figs. 3(b)-3(e) we show the excess charge-density $\Delta \rho$ in different parts of the system after transferring one electron for two different molecule-to-graphene distances. The excess charge-density $\Delta \rho$ is defined as $\rho_{\mathrm{CDFT}}-\rho_{\mathrm{DFT}}$, where $\rho_{\mathrm{DFT}}$ and $\rho_{\mathrm{CDFT}}$ are, respectively, the charge densities of the system before and after the charge transfer. Thus the portion of $\Delta \rho$ localized on the graphene sheet effectively corresponds to the image charge profile. Clearly due to the stronger Coulomb attraction, the image charge is more localized for $d=3.4 \AA$ than for $d=6.8 \AA$. At equilibrium for the $\mathrm{S}$ configuration, $d=3.25 \AA$, the charge transfer energy gap is calculated to be $8.91 \mathrm{eV}$, which is in good agreement (within $4 \%$ ) with the gap obtained by $G_{0} W_{0}$ [23]. In Table I, for the purpose of comparison, we have listed the charge transfer energies and

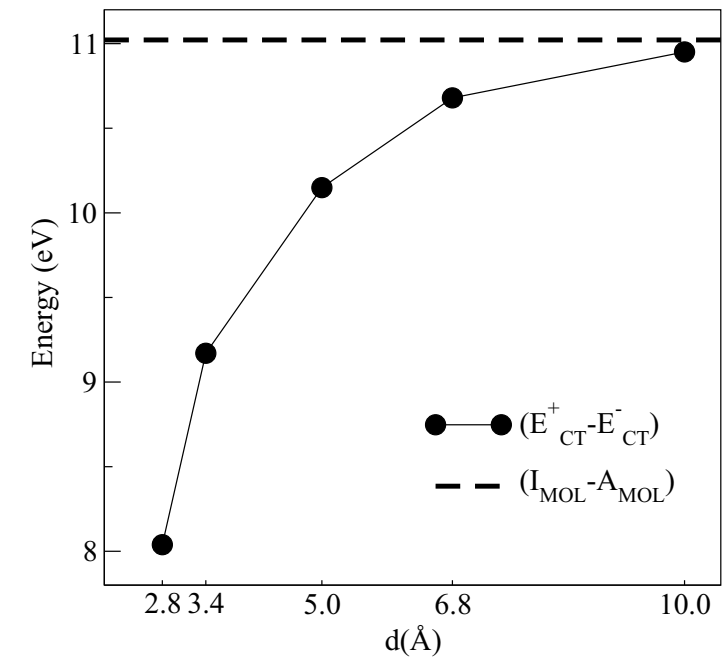

(a)

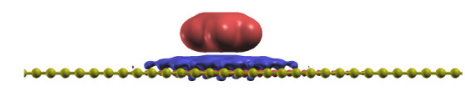

(b)

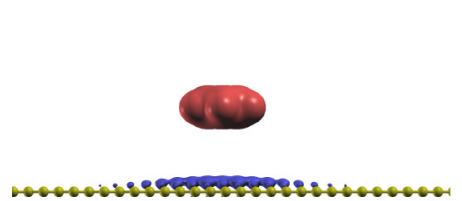

(d)

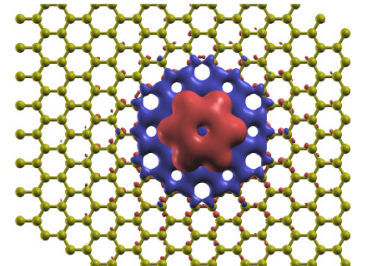

(c)

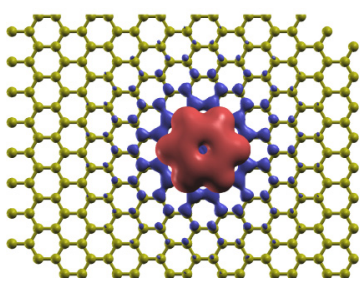

(e)
FIG. 3. Renormalization of the charge transfer gap as a function of the distance between the molecule and the graphene sheet (a). The dashed line denotes the difference between the IP and the EA calculated with the $\triangle \mathrm{SCF}$ method. (b) and (c) The excess charge in different parts of the system after the transfer of an electron from the molecule to the sheet for $d=3.4 \AA$. (d) and (e) The same plot for $d=6.8 \AA$. In both cases, red and blue denote positive and negative net charge, respectively. In practice the charge distribution over the graphene plane corresponds to the charge distribution of the image charge.

charge transfer gaps for two different heights, 3.4 and $6.8 \AA$, and in different configurations. The most notable feature is that for the case of a pristine graphene substrate the specific adsorption site plays little role in determining the charge transfer levels alignment.

\section{B. Benzene on graphene with Stone-Wales defect}

In general actual graphene samples always display lattice imperfections [27]. In order to determine the effect of such structural defects on the CT energies, we consider a reference system where a Stone-Wales (SW) defect (in which a single $\mathrm{C}-\mathrm{C}$ bond is rotated by $90^{\circ}$ ) is present in the graphene sheet. 
TABLE I. $E_{\mathrm{CT}}^{+}, E_{\mathrm{CT}}^{-}$, and $\Delta E_{\mathrm{CT}}=E_{\mathrm{CT}}^{+}-E_{\mathrm{CT}}^{-}$for various configurations of a benzene molecule on pristine and defective graphene. $\mathrm{H}$ and $\mathrm{S}$ denote adsorption of benzene on graphene in the hollow and stack configuration, respectively. $\mathrm{H}_{\mathrm{sw}-\mathrm{T}}$ and $\mathrm{H}_{\mathrm{sw}-\mathrm{A}}$ correspond to adsorption on graphene with SW defect, with the former corresponding to adsorption exactly on top of the defect and the latter corresponding to adsorption away from the site of the defect. The configurations $\mathrm{H}_{\mathrm{M} 1}$ and $\mathrm{H}_{\mathrm{M} 2}$ both correspond to adsorption of two benzene molecules in hollow configuration-one at height $3.4 \AA$ and another at a height $6.8 \AA$. While in $\mathrm{H}_{\mathrm{M} 1}$, the CT is calculated for the lower molecule, in $\mathrm{H}_{\mathrm{M} 2}$, the CT is calculated for the upper one. Finally, $\mathrm{H}_{\mathrm{L}}$ represents the case in which we have a layer of nonoverlapping benzenes adsorbed on graphene and one is interested in calculating the CT energy for one of them, which is placed in the hollow configuration.

\begin{tabular}{lcccc}
\hline \hline Configuration & $d(\AA)$ & $E_{\mathrm{CT}}^{+}(\mathrm{eV})$ & $E_{\mathrm{CT}}^{-}(\mathrm{eV})$ & $\Delta E_{\mathrm{CT}}(\mathrm{eV})$ \\
\hline $\mathrm{H}$ & 3.4 & 4.32 & -4.81 & 9.13 \\
$\mathrm{H}$ & 6.8 & 4.95 & -5.72 & 10.67 \\
$\mathrm{H}_{\mathrm{sw}-\mathrm{T}}$ & 3.4 & 4.30 & -4.78 & 9.08 \\
$\mathrm{H}_{\mathrm{sw}-\mathrm{A}}$ & 3.4 & 4.34 & -4.83 & 9.17 \\
$\mathrm{~S}$ & 3.4 & 4.33 & -4.81 & 9.14 \\
$\mathrm{~S}$ & 3.25 & 4.21 & -4.70 & 8.91 \\
$\mathrm{H}_{\mathrm{M} 1}$ & 3.4 & 4.12 & -4.76 & 8.88 \\
$\mathrm{H}_{\mathrm{M} 2}$ & 6.8 & 4.73 & -5.64 & 10.37 \\
$\mathrm{H}_{\mathrm{L}}$ & 3.4 & 4.27 & -4.62 & 8.89 \\
\hline \hline
\end{tabular}

We have then calculated $E_{\mathrm{CT}}^{ \pm}$for two different positions of the molecule with respect to the defect on the sheet, namely the $\mathrm{H}_{\mathrm{sw}-\mathrm{T}}$ position, in which the molecule is placed right above the defect and the $\mathrm{H}_{\mathrm{sw}-\mathrm{A}}$ position, in which it is placed above the sheet far from the defect (see Fig. 1).

Our findings are listed in Table I, where we report the charge transfer energies for both configurations, assuming the molecule is kept at the same distance from the graphene sheet. From the table it is evident that the structural change in graphene due to presence of such defect does not alter the charge transfer energies of the molecule. This is because the image charge distribution on graphene is little affected by presence of the SW defect. In addition, the density of states (DOS) of graphene remains almost completely unchanged near its Fermi energy after introducing such defect as can be seen in Fig. 4, which shows that the partial density of states (PDOS) of the atoms forming the SW defect has no significant presence near the Fermi level. Thus, after the charge transfer, the electron added to (or removed from) the graphene sheet has the same energy that it would have in the absence of the defect, i.e., it is subtracted (added) from a region of the DOS where there is no contribution from the SW defect. In this context it is noteworthy that a $G_{0} W_{0}$ study [23] has concluded that altering the structure of pristine graphene by introducing dopant (which raises the Fermi level of graphene by $1 \mathrm{eV}$ ) also has minor effect on the QP gap of benzene, reducing it by less than $3 \%$.

\section{Multiple benzene molecules adsorbed on graphene}

In real interfaces between organic molecules and a substrate, molecules usually are not found isolated but in proximity to others. It is then interesting to investigate the effects that the presence of other benzene molecules produce of the charge transfer energies of a given one. To this end we select

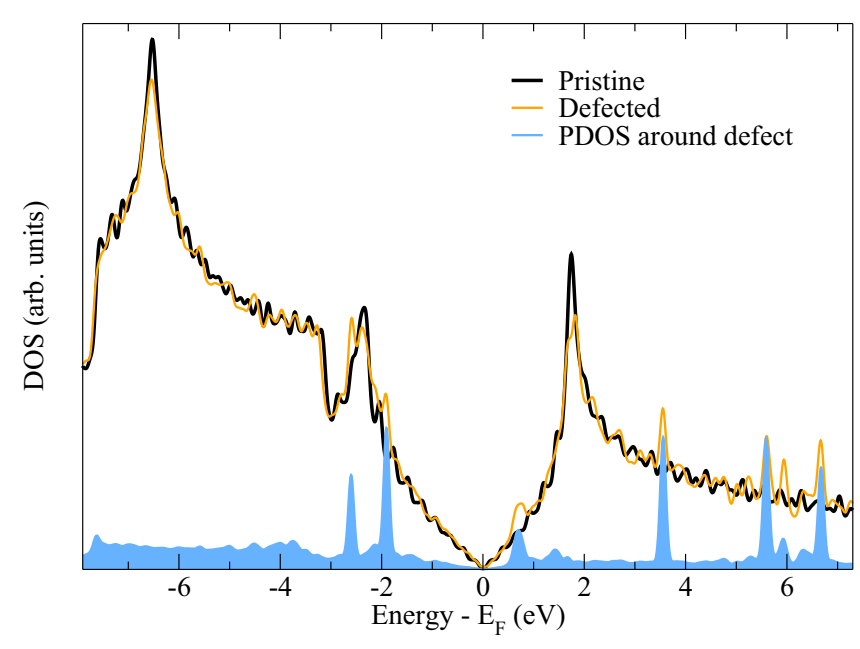

FIG. 4. DOS of pristine graphene (black line), DOS of a graphene sheet with one SW defect (orange line), and PDOS for the atoms adjacent to the SW defect (blue shade). The latter has been multiplied by 3 for better visibility. These calculations are performed with a supercell of 200 atoms containing one SW defect.

three representative configurations. In the first one $\mathrm{H}_{\mathrm{M} 1}$, the graphene sheet is decorated with two benzene molecules, one at $3.4 \AA$ while the other is placed above the first at $6.8 \AA$ from the graphene plane. Note that we have chosen the distances of 3.4 and $6.8 \AA$ so that the new results can be readily compared with those obtained in the previous section for a single benzene molecule. This means that the geometry of such double-benzene structure has not been fully relaxed. We then calculate the charge transfer energies of the middle benzene (the one at $3.4 \AA$ from the sheet). The excess charge on different parts of the system (image charge), after transferring one electron to the sheet, is displayed in Figs. 5(a) and 5(b). The second configuration $\mathrm{H}_{\mathrm{M} 2}$, is identical to the first one but now we calculate the charge transfer energies of the molecule, which is farther away from the graphene sheet, namely at a distance of $6.8 \AA$. For this configuration, the excess charge after a similar charge transfer is shown in Figs. 5(c) and 5(d). In the third configuration $\mathrm{H}_{\mathrm{L}}$, we arrange multiple benzene molecules in the same plane. The molecules are in close proximity with each other although their atomic orbitals do not overlap. Charge transfer energies are then calculated with respect to one benzene molecule keeping the others neutral and an isovalue plot for similar charge transfer is shown in Figs. 5(e) and 5(f).

The charge transfer energies calculated for these three configurations are shown in Table I. If one compares configurations where the molecule is kept at the same distance from the graphene plane, such as the case of $\mathrm{H}(d=3.4 \AA)$, $\mathrm{H}_{\mathrm{M} 1}$, and $\mathrm{H}_{\mathrm{L}}$ or of $\mathrm{H}(d=6.8 \AA)$ and $\mathrm{H}_{\mathrm{M} 2}$, it appears clear that the presence of other molecules has some effect on the charge transfer energies. In particular, we observe than when other molecules are present both $E_{\mathrm{CT}}^{+}$and $E_{\mathrm{CT}}^{-}$get more shallow, i.e., their absolute values are reduced. Interestingly the relative reduction of $E_{\mathrm{CT}}^{+}$and $E_{\mathrm{CT}}^{-}$depends on the details of the positions of the other molecules (e.g., it is different for $\mathrm{H}_{\mathrm{M} 1}$ and $\mathrm{H}_{\mathrm{L}}$ ) but the resulting renormalization of the HOMO-LUMO 


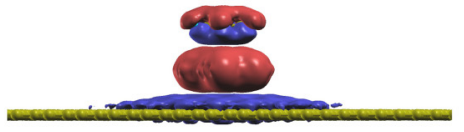

(a)

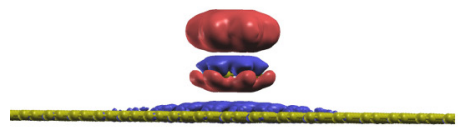

(c)

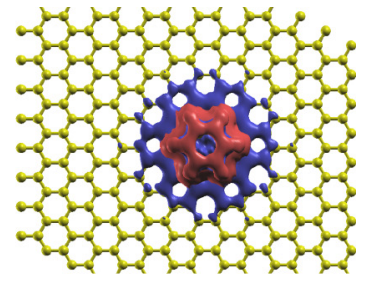

(b)

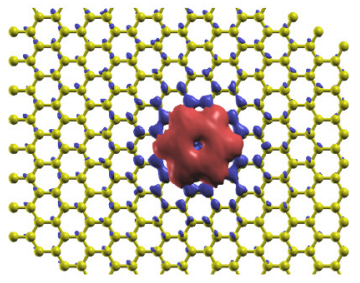

(d)

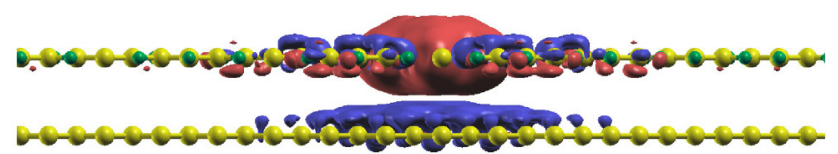

(e)

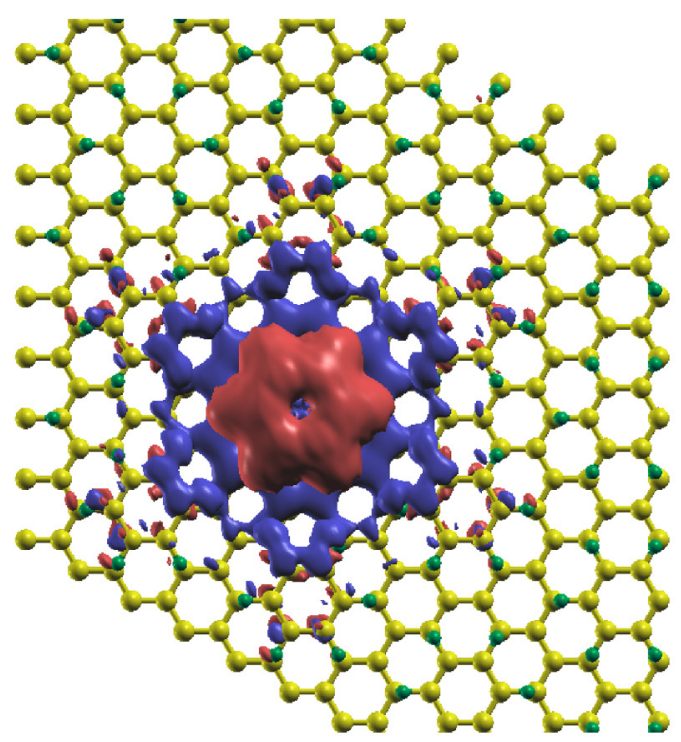

(f)

FIG. 5. Image charge formation for various configurations in which more than one benzene molecule is present. (a) and (b) The side and top view of the isovalue of $\Delta \rho(\mathbf{r})$ after transferring one electron from the molecule to the sheet for the $\mathrm{H}_{\mathrm{M} 1}$ case. (c) and (d) and (e) and (f) Plots of the same quantity for the cases of $\mathrm{H}_{\mathrm{M} 2}$ and $\mathrm{H}_{\mathrm{L}}$, respectively.

gap is essentially identical [about $250 \mathrm{meV}$ when going from $\mathrm{H}(d=3.4 \AA)$ to either $\mathrm{H}_{\mathrm{M} 1}$ or $\left.\mathrm{H}_{\mathrm{L}}\right]$.

This behavior can be explained in terms of a simple classical effect. Consider the case of $\mathrm{H}_{\mathrm{M} 1}$ for example. When one

transfers an electron from the middle benzene to the graphene sheet the second benzene molecule, placed above the first, remains neutral but develops an induced charge dipole. The moment of such dipole points away from the charged benzene and lowers the associated electrostatic potential. Importantly, also the potential of graphene will be lowered. However, since the potential generated by an electrical dipole is inversely proportional to the square of distance, the effect remains more pronounced at the site of the middle benzene than at that of the graphene sheet. A similar effect can be observed for an electron transfer from the graphene sheet to the middle benzene and for the $\mathrm{H}_{\mathrm{L}}$ configuration.

In the case of $\mathrm{H}_{\mathrm{M} 2}$, the system comprising the topmost benzene (from which we transfer charge) and the graphene plane can be thought of as a parallel-plate capacitor. The work $W$ done to transfer a charge $Q$ from one plate to the other is $W=Q^{2} / 2 C$, where $C$ is the capacitance, which in turn is proportional to the dielectric constant of the medium enclosed between the plates. Hence, at variance with the case of $\mathrm{H}(d=$ $6.8 \AA)$, the space in between the molecule and the graphene sheet is occupied by a molecule with finite dielectric constant and not by vacuum. This results in a reduction of $W$, so that the charge transfer energies for $\mathrm{H}_{\mathrm{M} 2}$ are smaller than those for $\mathrm{H}(d=6.8 \AA)$.

\section{Classical electrostatic approximation}

Finally, we show that our calculated energy level alignment can be obtained from a classical electrostatic model. If one approximates the transferred electron as a point charge and the substrate where the image charge forms as an infinite sheet of relative permittivity $\epsilon$ then, for a completely planar distribution of the bound surface charge, the work done by the induced charge to take an electron from the position of the molecule (at a distance $d$ ) to infinity is

$$
W=-\frac{1}{4 \pi \epsilon_{0}}\left(\frac{\epsilon-1}{\epsilon+1}\right) \frac{q^{2}}{4 d} .
$$

Hence, this electrostatic approximation predicts that the presence of the substrate lowers the LUMO of the molecule by $-\frac{1}{4 \pi \epsilon_{0}}\left(\frac{\epsilon-1}{\epsilon+1}\right) \frac{q^{2}}{4 d}$ with respect to the corresponding gas-phase value. However, the actual image charge is not strictly confined to a $2 \mathrm{D}$ plane but instead spills out over the graphene surface. We can account for such nonplanar image charge distribution by introducing a small modification to the above expression [28] and write the LUMO at a height $d$ as

$$
\operatorname{LUMO}(d)=\operatorname{LUMO}(\infty)-\frac{1}{4 \pi \epsilon_{0}}\left(\frac{\epsilon-1}{\epsilon+1}\right) \frac{q^{2}}{4\left(d-d_{0}\right)},
$$

where $d_{0}$ is the distance between the center of mass of the image charge and the substrate plane and $\operatorname{LUMO}(\infty)$ is the gas-phase LUMO (the electron affinity). A similar argument for the HOMO level shows an elevation of same magnitude due to the presence of the substrate. In Fig. 6 we plot the charge transfer energies and show that they compare quite well with the curves predicted by the classical model by using an effective dielectric constant of 2.4 for graphene [29]. When drawing the classical curves we have used an approximate value $d_{0}=1.7 \AA$, which provides an excellent estimate for smaller distances $d$. It is worth noting that for larger distances, 


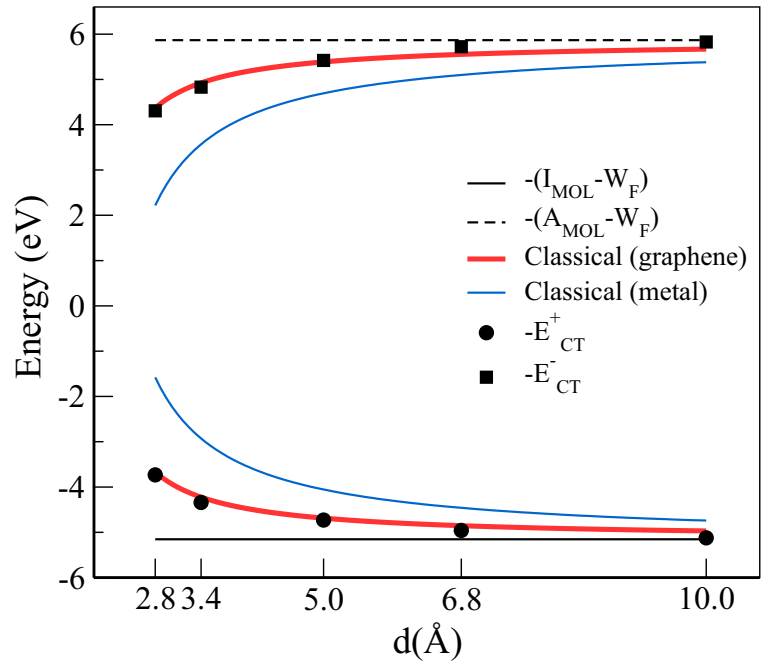

FIG. 6. $-E_{\mathrm{CT}}^{+}$(circles) and $-E_{\mathrm{CT}}^{-}$(squares) calculated for different molecule-to-substrate distances. The CDFT results are seen to agree well with the classically calculated curve given in red. The horizontal lines mark the same quantities for an isolated molecule (gas-phase quantities). The continuous blue line shows the position of the classically calculated level curve for adsorption on a perfect metal $\epsilon=\infty$.

though the actual value of $d_{0}$ should be much less, the overall effect of $d_{0}$ is very small and almost negligible. In the same graph we have also plotted the classical curves corresponding to benzene on a perfectly metallic $(\epsilon=\infty)$ surface. This shows that the level renormalization of benzene for physisorption on graphene is significantly different from that on a perfect metal, owing to the different screening properties of graphene.

\section{CONCLUSION}

We have used CDFT as implemented in the SIESTA code to calculate the energy level alignment of a benzene molecule adsorbed on a graphene sheet. In general the charge transfer energies depend on the distance between the molecule and the graphene sheet, and this is a consequence of the image charge formation. Such an effect cannot be described by standard Kohn-Sham DFT, but it is well captured by CDFT, which translates a quasiparticle problem into one about energy differences. With CDFT we have simulated the energy level renormalization as a function of the moleculeto-graphene distance. The charge transfer energies for an infinite separation agree well with experimental data about the ionization potential and the electron affinity. Furthermore, an excellent agreement is also obtained with $G W$ calculations at typical bonding distances. Since CDFT is computationally inexpensive we have been able to study the effects arising from bonding the molecule to a graphene structural defect and from the presence of other benzene molecules. We have found that a Stone-Wales defect does not affect the energy level alignment since its electronic density of state has little amplitude at the graphene Fermi level. In contrast the charge transfer energies change when more then a molecule is present. All our results can be easily rationalized by a simple classical electrostatic model describing the interaction of a pointlike charge and a uniform planar charge distribution. This, at variance to the case of a perfect metal, takes into account the finite dielectric constant of graphene.

\section{ACKNOWLEDGMENTS}

This work is supported by the European Research Council, Quest project. Computational resources have been provided by the supercomputer facilities at the Trinity Center for High Performance Computing (TCHPC) and at the Irish Center for High End Computing (ICHEC). Additionally, the authors would like to thank Dr. Ivan Rungger and Dr. A. M. Souza for helpful discussions.
[1] C. Dimitrakopoulos and P. Malenfant, Adv. Mater. 14, 99 (2002).

[2] J. W. Gadzuk, Phys. Rev. Lett. 76, 4234 (1996).

[3] J. Repp, Phys. Rev. Lett. 94, 026803 (2005).

[4] N. Koch, ChemPhysChem. 8, 1438 (2007).

[5] S. Sanvito, Chem. Soc. Rev. 40, 3336 (2011).

[6] P. Hohenberg and W. Kohn, Phys. Rev. 136, B864 (1964).

[7] J. F. Janak, Phys. Rev. B 18, 7165 (1978).

[8] A. D. Becke, J. Chem. Phys. 98, 1372 (1993).

[9] C. D. Pemmaraju, T. Archer, D. Sánchez-Portal, and S. Sanvito, Phys. Rev. B 75, 045101 (2007).

[10] A. Filippetti, C. D. Pemmaraju, S. Sanvito, P. Delugas, D. Puggioni, and V. Fiorentini, Phys. Rev. B 84, 195127 (2011).

[11] S. Refaely-Abramson, S. Sharifzadeh, N. Govind, J. Autschbach, J. B. Neaton, R. Baer, and L. Kronik, Phys. Rev. Lett. 109, 226405 (2012).

[12] D. A. Egger, S. Weissman, S. Refaely-Abramson, S. Sharifzadeh, M. Dauth, R. Baer, S. Kümmel, J. B. Neaton, E. Zojer, and L. Kronik, J. Chem. Theory Comput. 10, 1934 (2014).
[13] K. S. Thygesen and A. Rubio, Phys. Rev. Lett. 102, 046802 (2009).

[14] M. S. Hybertsen and S. G. Louie, Phys. Rev. B 34, 5390 (1986).

[15] J. B. Neaton, M. S. Hybertsen, and S. G. Louie, Phys. Rev. Lett. 97, 216405 (2006).

[16] Q. Wu and T. Van Voorhis, Phys. Rev. A 72, 024502 (2005).

[17] A. M. Souza, I. Rungger, C. D. Pemmaraju, U. Schwingenschloegl, and S. Sanvito, Phys. Rev. B 88, 165112 (2013).

[18] A. C. Ferrari et al., Nanoscale 7, 4598 (2015).

[19] Y. Wang, J. Torres, A. Stieg, S. Jiang, M. Yeung, Y. Rubin, S. Chaudhuri, X. Duan, and R. Kaner, ACS Nano 9, 9486 (2015).

[20] A. AlZahrani, Appl. Surf. Sci. 257, 807 (2010).

[21] S. D. Chakarova-Käck, E. Schröder, B. I. Lundqvist, and D. C. Langreth, Phys. Rev. Lett. 96, 146107 (2006).

[22] Y.-H. Zhang, K.-G. Zhou, K.-F. Xie, J. Zeng, H.-L. Zhang, and Y. Peng, Nanotechnology 21, 065201 (2010). 
[23] V. Despoja, I. Lončarić, D. J. Mowbray, and L. Marušić, Phys. Rev. B 88, 235437 (2013).

[24] J. M. Soler, E. Artacho, J. D. Gale, A. García, J. Junquera, P. Ordejón, and D. Sánchez-Portal, J. Phys.: Condens. Matter 14, 2745 (2002).

[25] P. Clare and D. B. Sowerby, J. Inorg. Nucl. Chem. 43, 477 (1981).
[26] P. Burrow, J. A. Michejda, and K. D. Jordan, J. Chem. Phys. 86, 9 (1987).

[27] F. Banhart, J. Kotakoski, and A. V. Krasheninnikov, ACS Nano 5, 26 (2001).

[28] N. D. Lang and W. Kohn, Phys. Rev. B 3, 1215 (1971).

[29] B. Wunsch, T. Stauber, F. Sols, and F. Guinea, New J. Phys. 8, 318 (2006). 